\title{
TAIWÁN EN 2017 RETOS DEL GOBIERNO TAIWANÉS
}

Marisela Connelly

El Colegio de México

\section{INTRODUCCIÓN}

En enero de 2016 se llevaron a cabo elecciones presidenciales y legislativas en Taiwán. Por primera vez en la isla, una mujer, Cai Yingwen, del Partido Democrático Progresista (PDP), ganó la presidencia con 56\% de los votos. En la legislatura, el PDP ganó 68 asientos de los 113 que componen este órgano de gobierno. El Guomindang se quedó con sólo 35; el Partido Nuevo Poder que promueve la identidad de Taiwán como país, se quedó con 5, y el Partido Primero el Pueblo, de James Soong, con 3.

Cai Yingwen prometió en su campaña sanear la economía, incrementar el presupuesto para defensa y bienestar social, diversificar los socios comerciales, entre otros. Como sucede de manera frecuente, una vez en el poder, las cosas se complican por la inexperiencia y por la toma de decisiones, que al final no satisfacen ni a los propios seguidores. La situación en el estrecho de Taiwán no ha mejorado y sigue el impasse pues Cai se niega a reconocer el Consenso de 1992, requisito indispensable que exige la República Popular China para entablar un diálogo con la isla. 


\section{POLÍTICA ECONÓMICA}

Cai Yingwen y Lin Chuan, entonces primer ministro, pusieron atención en cinco industrias: energía verde, maquinaría inteligente, expansión y mejoramiento de los servicios de internet, biomedicina y defensa. Lo que pretendían hacer era vincularlas a localidades específicas. En Taizhong se estableció el plan para la maquinaria inteligente. En Tainan, lo relativo a energía verde y en Taoyuan el desarrollo de internet para optimización de protocolos y funciones. Estas localidades están gobernadas por alcaldes del partido en el poder, el Partido Democrático Progresista. El desarrollo de energía limpia es un punto importante en la agenda de Cai dado que pretende eliminar la dependencia en energía nuclear de manera gradual. Para lograrlo, Cai ha firmado contratos con firmas tanto taiwanesas como extranjeras para proyectos solares. En el mes de marzo, el Yuan Ejecutivo anunció un plan de infraestructura de ocho años con una inversión de USD \$1 650 millones para inversión en energía verde. La meta es expandir la energía renovable de $5 \%$ en 2016 a $20 \%$ en $2025 .{ }^{1}$

El Consejo para el Desarrollo Nacional estableció la Agencia de Desarrollo del Valle Asiático de Silicio en la ciudad de Taoyuan para el desarrollo del internet.

En lo relativo a defensa, el gobierno de Cai firmó un contrato con la Corporación de Construcción Naval China para el diseño y producción de un submarino que se desarrollará en la ciudad sureña de Gaoxiong, y otro con la Corporación para el Desarrollo de la Industria Aeroespacial para la fabricación de un jet.

Lin Chuan propuso crear un presupuesto especial de USD\$29 mil millones para un proyecto de construcción de infraestructura por un periodo de ocho años. Un componente de este proyecto es el plan de energía verde, además del desarrollo de ferrocarriles. La idea también es atraer a talento tanto extranjero como taiwanés para que participen en estos proyectos.

La administración del PDP ha estado trabajando arduamente para lograr que la economía se robustezca; se estimaba que la economía crecería en 2017 sólo 1.9\%, pero dada la tendencia en la economía mundial de crecimiento positivo, se espera que la economía de

${ }^{1}$ David G. Brown, “Governing Taiwan is not easy: President Tsai Ing-wen First Year”, Brookings, mayo de 2017; <http://www. Brookings.edu> $>$. Consultado el 24 de junio de 2017. 
la isla crezca 2 o 2.5\%. Los productos exportados por Taiwán están enfrentándose a una competencia cada vez mayor en el sector de electrónicos desarrollados por otros países. En el periodo de enero a septiembre de 2017, las exportaciones de Taiwán crecieron 14.3\% comparado con el mismo lapso del año anterior, y las importaciones aumentaron $14.7 \%$ en el mismo periodo, de acuerdo con el Ministerio de Asuntos Económicos. La posición de Cai de tratar de disminuir la dependencia de la economía taiwanesa con respecto a China no ha logrado mucho éxito. Las exportaciones de Taiwán hacia China constituyen $40 \%$ del total. Por otro lado, Cai Yingwen ha tratado de diversificar sus relaciones comerciales con el fin de romper la dependencia económica con China. Los países de la ANSEA constituyen el segundo mercado para Taiwán, con 18\% de sus exportaciones en 2016. Le sigue Estados Unidos con $12 \%$ y después Japón con $7 \%$. Cai ha tratado de ayudar a sus compañías establecidas en los países de la ANSEA que se han visto limitadas por las presiones de Beijing a esos países y porque la isla no tiene relaciones diplomáticas con ellos. Los empresarios

taiwaneses tienen una vinculación muy estrecha y de años con China. No obstante, esta relación está sufriendo cambios cualitativos debido a los avances tecnológicos experimentados en el continente, de tal forma que ya es más competitiva que complementaria. Las empresas chinas están logrando productos con la misma sofisticación que las taiwanesas. No obstante, las empresas taiwanesas tienen la ventaja de sus contratos con grandes compañías como Apple a las que les suministra componentes.

\section{SiTUACIÓN POLÍTICA}

El estilo de gobernar de Cai Yingwen muestra una tendencia hacia la concentración de toma de decisiones en la presidencia, pidiendo a los ministros presentar sus reportes de trabajo directamente a ella y no al primer ministro, como se había hecho en gobiernos anteriores. Desde su campaña estaba pensando en llevar a cabo una reforma política. El establecimiento de un proceso transparente, además de la reforma de pensiones y la judicial. Cai ha logrado abrir el sistema haciéndolo más inclusivo y uno de los elementos claves ha sido el cambio en el proceso legislativo. Los debates legislativos son más ágiles y se trasmiten por televisión.

En cuanto a la reforma de pensiones, ha sido difícil y ha estado sujeta a constantes debates y discusiones. La reforma afecta los intereses de los maestros, militares y miembros 
del servicio civil que han gozado de pensiones generosas. Tanto el Yuan Ejecutivo como el Yuan de Exámenes y los partidos de oposición han presentado sus versiones legislativas. Durante las discusiones en el Yuan Legislativo, los diferentes grupos, con posiciones contrarias, han llegado a las agresiones físicas. Cai ha declarado que pese a ese ambiente de confrontación, la legislación debe ser aprobada. El público en general ha sido muy crítico de la posición del gobierno.

La reforma judicial ha sufrido tropiezos con el nombramiento de los comités que se establecerían para propuestas y discusión. Los desacuerdos y las renuncias de miembros de esos comités no han hecho posible un avance. ${ }^{2}$

La administración de Cai Yingwen se ha enfrentado al Guomindang (GMD) por la promoción de una propuesta de los legisladores del PDP, para realizar una investigación sobre los activos financieros del GMD que llevó a la congelación de sus activos. Todo ello produjo un enrarecimiento del ambiente político.

Los trabajadores taiwaneses, por su parte, presionaron al gobierno para que restaurara los días festivos suprimidos y la jornada de trabajo de 40 horas semanales. En los países de la OCDE se trabajan al año 1771 horas, en tanto que en Taiwán se trabajan 2 134. Los empresarios mostraron su oposición a la reducción de horas y al restablecimiento de días festivos. El PDP en el Yuan Legislativo logró consenso para que se aprobara reducir la jornada de trabajo pero sin la restauración de días festivos. Ni los trabajadores ni los empresarios quedaron satisfechos.

El otro asunto que también fue muy controvertido fue el relativo a la justicia transicional para los pueblos indígenas. Cai se disculpó con ellos por los malos tratos que habían recibido por tantos años. Estos pueblos se sintieron apoyados por la presidenta y empezaron a reclamar las tierras que les habían quitado. La posición del gobierno fue la de negociar con respecto a tierras de indígenas que ahora estaban en posesión del gobierno, pero no respecto a las que estaban en manos privadas. Está posición fue muy criticada por la opinión pública. ${ }^{3}$ La presidenta Cai también dio impulso a la lengua hakka lanzando una estación de radio en ese idioma. Además, dijo que en lugares en donde este dialecto se hable

\footnotetext{
${ }^{2}$ David G. Brown, op. cit.

${ }^{3}$ Ibidem.
} 
por más de un tercio de la población, las escuelas primarias y secundarias deberán enseñarla en forma obligatoria. ${ }^{4}$

El 4 de septiembre el PM Lin Chuan presentó su renuncia diciendo que ya había cumplido con su cometido de asegurar la transición de poder de manera suave, consolidar las promesas de campaña de Cai y dejar las bases para el desarrollo nacional. Lin, de 65 años, fue criticado por llevar a cabo las reformas que afectaron a trabajadores, empleados, servidores civiles retirados y del servicio militar. Las protestas populares hicieron que la popularidad de Cai bajara de $70 \%$, cuando tomó posesión de su cargo, a $28 \%$ en septiembre. ${ }^{5}$

El 8 de septiembre, el nuevo PM, William Lai Qinde, tomó posesión de su cargo, lo mismo que los 44 miembros de su gabinete. Señaló que su prioridad era fortalecer la economía local, y facilitar la transformación económica y política de la isla. El 7 de septiembre, Lai renunció a su puesto de alcalde de Tainan, puesto que tenía desde 2010. Lai estuvo 11 años como legislador. ${ }^{6}$ No obstante que el nuevo primer ministro hizo pública su posición de apoyo a la independencia, también postuló el eslogan "ser amigable con China y amar a Taiwán” (親中愛台).

\section{GUOMINDANG}

En mayo, el exvicepresidente Wu Denyi, taiwanés, fue electo como nuevo presidente del partido, con 52\% del voto, ganándole a la presidenta Hong Xiuzhu.

En junio, Hong visitó Beijing y fue recibida con alfombra roja, tuvo pláticas con el ministro de Asuntos de Taiwán, Zhang Zhijun, en Nanjing. Se entrevistó también con Yu Zhengsheng, miembro del Comité Permanente del Politburó en el noveno Foro del Estrecho en Fujian.

En el Congreso del GMD en agosto, Wu tomó posesión de su cargo. Se comprometió a seguir una política de partido que privilegie las buenas relaciones a través del estrecho,

${ }^{4}$ Taiwan News, 24 de junio de 2017. Disponible en: $<\mathrm{http}: / /$ www.taiwannews.com.tw $>$. Consultado el 1 de julio de 2017.

${ }_{6}^{5}$ South China Morning Post, 4 de septiembre de 2017. Taipei Times, 5 de septiembre de 2017.

${ }^{6}$ Taiwan Today, 8 de septiembre de 2017. 
siguiendo lo establecido en la declaración conjunta Lian Zhan-Hu Jintao de 2005 y las iniciativas de Ma Yingjiu.

El GMD está sumido en la disensión interna y problemas financieros. Hong Xiuzhu ha estado en desacuerdo con el grupo parlamentario del PDP en el Yuan Legislativo sobre prácticas políticas.

Finalmente, el expresidente Ma Yingjiu fue liberado de los cargos que le habían hecho en 2013 de revelar secretos y de instar a un fiscal a revelar información secreta respecto a un caso judicial. $^{7}$

\section{SitUACIÓN EN EL ESTRECHO DE TAIWÁN: NO ACEPTACIÓN DEL “PRINCIPIO DE UNA SOLA CHINA”, NO DIÁLOGO}

Desde el inicio de su gobierno Cai Yingwen explicó que las relaciones en el estrecho de Taiwán seguirían los parámetros marcados por la Constitución de la República de China, el Acta que Gobierna las Relaciones entre los Pueblos del Área de Taiwán y el Área Continental. Pidió al gobierno chino que aceptara entablar un diálogo constructivo. Pero, bajo esas premisas, lo único que hubo del otro lado fue crítica a lo declarado por la presidenta.

En el gobierno de Xi Jinping, la política hacia Taiwán se ha vuelto rígida y asertiva. Ello se debe a la dependencia tan grande de la economía de la isla hacia el continente, proceso que se aceleró en los ocho años de gobierno de Ma Yingjiu. El otro punto es el relativo a la posición que ahora ocupa China en el este de Asia y en el mundo que muestra la fortaleza del país, y que ha hecho pensar a sus líderes en la necesidad de solucionar, lo más rápido posible, el asunto de la unificación. Se ha marcado como fecha tentativa para la reunificación 2049, año en que se cumplen 100 años del establecimiento de la RPCh.

La táctica del Frente Unido puesta en práctica por el gobierno de Beijing para utilizar todos los medios a su alcance, mostrar su fuerza y compromiso con su política de unificación mostró, muy pronto, resultados. Desde junio de 2016, el gobierno chino suspendió toda comunicación con Taipéi. Restringió los viajes de turistas del continente hacia Taiwán

\footnotetext{
${ }^{7}$ South China Morning Post, 25 de agosto de 2017.
} 
afectando seriamente la industria turística de la isla y ha llevado a los trabajadores de la industria turística a realizar protestas en la capital de la isla. El número de turistas chinos se ha reducido de manera drástica. Se estima que el déficit de visitantes chinos este año llegará a $37.7 \%$ con respecto al año anterior. Las empresas del sector servicios han sentido el impacto.

La participación de Taiwán en la Asamblea de la Organización Mundial de la Salud, que desde 2009 contaba con la asistencia de un representante de la isla, fue bloqueada nuevamente. En mayo de 2017, Taiwán no fue invitado a la Asamblea de la Organización Mundial de la Salud. Lo mismo ocurrió en la Organización Internacional de Aviación Civil, a cuya asamblea anterior había asistido un representante de Taipéi. La oficina de Cai sacó un comunicado que decía que reiteraba la soberanía de la República de China y el hecho de que Taiwán no era una provincia china. El ministro de Relaciones Exteriores declaró que la aceptación del principio de "una China" no era requisito para ser observador de la OMS. El ministro de Salud de Taiwán, Chen Shizhong, viajó a Ginebra para tener conversaciones con algunos de sus colegas. A la reunión de los líderes de los países pertenecientes al grupo de Cooperación Económica Asia Pacífico asistió en representación de Cai, James Soong, del Partido Primero el Pueblo. Xi Jinping trató de que no tuviera contacto con Shinzo Abe, de Japón, pero fue en vano. Abe se entrevistó con Soong. ${ }^{8}$

En una entrevista para el diario Lianhe Bao (Diario Unido) el 3 de mayo, Cai propuso: que los dos lados reconocieran que existía una nueva situación, nuevos planteamientos y, por tanto, había que desarrollar nuevas formas de interacción que dejaran atrás los métodos burocráticos e ineficientes 新情勢、新問卷、新模式). Posteriormente, Cai explicó que la nueva situación estaba dada por el establecimiento y desarrollo de la democracia en Taiwán. Pidió al presidente Xi Jinping trabajar en conjunto por relaciones estables que beneficien a los dos lados del estrecho. El viceministro de Asuntos Continentales de Taiwán defendió la posición del gobierno de preservar la democracia, desarrollar la identidad taiwanesa y esforzarse por llegar a ser un "país normal". 9

\footnotetext{
${ }^{8}$ Salvatore Babones, "Taiwan is at the center of Asia's Economy, but on the margins of its meetings", en: $<$ http://www.forbes,com>. Consultado el 12 de noviembre de 2017.

${ }^{9}<$ http://www.mac.gov.tw/en/News_Content.aspx?n=BEC36A4A0BB0663C\&sms=BF821F021B282 251\&s=CA5D6FE2FD804167>. Consultado el 3 de agosto de 2017.
} 
Pero el presidente Xi, en agosto, en su discurso para conmemorar el 90 aniversario de la fundación del Ejército de Liberación Popular señaló que no permitirían que se perdiera ni una sola parte del territorio chino, nada que afectara la soberanía, la seguridad y el desarrollo. Reiteró esa posición en su discurso en el Décimo Noveno Congreso del PCCh, en donde dijo que se opondrían de manera resoluta a la independencia de Taiwán. Buscarían la resolución de la cuestión de Taiwán para así lograr la completa unificación del país. Defenderían los principios de unificación pacífica "un país, dos sistemas". Trabajarían para el desarrollo pacífico de las relaciones en el estrecho y así avanzar en el proceso de unificación pacífica. Xi señaló que el Consenso de 1992 encarnaba el "principio de una China" y definía la naturaleza de las relaciones en el estrecho. Con la aceptación de estos principios, enfatizó, cualquier partido en Taiwán podría tener intercambios con el continente. A cambio, el gobierno chino ofrecía respetar el sistema social y modo de vida en la isla y compartir las oportunidades de desarrollo, de tal manera que los taiwaneses puedan tener el mismo trato que los locales en estudio, trabajo, negocios. Pero advirtió que de ningún modo se permitirían movimientos separatistas que condujeran a la independencia de Taiwán. ${ }^{10}$

La reacción del Ministerio de Asuntos Continentales de Taiwán fue de rechazo a lo dicho por Xi Jinping. Insistió en que la República de China era un país soberano y sus ciudadanos tenían el derecho de decidir sobre el futuro de la isla y el desarrollo de las relaciones en el estrecho. Pidió al gobierno de Beijing que asumiera el hecho de que para los taiwaneses era difícil aceptar la fórmula "un país, dos sistemas" y el "principio de una sola China". ${ }^{11}$

Poniendo en práctica las políticas hacia los taiwaneses y así atraer su simpatía, fue anunciado que los residentes en el continente podrían presentar sus solicitudes para trabajar en instituciones públicas en seis regiones a nivel provincial; los académicos taiwaneses que trabajaran en China podrían solicitar su participación en proyectos estatales incluyendo los relativos al Fondo Nacional de Ciencias Sociales. Asimismo, los estudiantes taiwaneses se

${ }^{10}$ Xi Jinping, "Secure a Decisive Victory in Building a Moderately Prosperous Society in All Respects and Strive for the Great Success of Socialism with Chinese Characteristics for a New Era". Discurso en el Décimo Noveno Congreso del Partido Comunista Chino. 18 de octubre de 2017, p. 51.

${ }^{11}$ Taipei Times, 19 de octubre de 2017. 
verían beneficiados con las medidas tomadas por el Ministerio de Educación tendientes a facilitar que estos estudiantes encuentren trabajo en el país al finalizar sus estudios. ${ }^{12}$

Al mismo tiempo, el Gobierno de Beijing está tratando de establecer un diálogo con los representantes de gobiernos locales de la isla. En julio, Ge Wenre, alcalde de Taipéi, visitó Shanghái para asistir al Foro Taipéi-Shanghái. El ministro de la Oficina de Asuntos de Taiwán comentó que China daba la bienvenida a los taiwaneses, siempre y cuando trabajaran por mejorar las relaciones en el estrecho de Taiwán. Por su parte, Ge Wenre señaló que podría romperse el actual impasse si los esfuerzos se basaran en el concepto de que los dos lados del estrecho pertenecen a una sola familia. ${ }^{13}$

\section{RELACIONES CON EL EXTERIOR}

El nuevo gobierno taiwanés ha hecho esfuerzos por acercarse a Estados Unidos después del triunfo de Donald Trump en las elecciones presidenciales. El 2 de diciembre de 2016, Cai Yingwen llamó por teléfono a Trump para felicitarlo por su triunfo. Trump, a su vez, felicitó a Cai por haber ganado las elecciones presidenciales en la isla. Hablaron sobre asuntos económicos y de seguridad. Este hecho no tenía precedente y el gobierno chino inmediatamente declaró por medio del portavoz del Ministerio de Relaciones Exteriores, Geng Shuang, que Washington debía respetar la política de "una sola China". Finalmente, el gobierno de Estados Unidos dejó en claro que no había cambio en su política hacia Taiwán. En febrero de 2017, Trump habló por teléfono con Xi Jinping y le reiteró el respeto a la política de "una sola China". ${ }^{14}$

En julio de 2017, el Departamento de Estado de Estados Unidos aprobó la venta de un nuevo paquete de armas a Taiwán que incluía misiles de alta velocidad, torpedos lanzados de submarinos, entre otros. ${ }^{15}$ El paquete necesitaba la aprobación del Congreso. El secretario de Estado Rex Tillerson anteriormente había anunciado que Estados Unidos continuaría cumpliendo sus compromisos con Taiwán. Por su parte, el Comité de Servicios de la Marina

\footnotetext{
${ }^{12}$ Xinhua, 12 de mayo de 2017.

${ }^{13}$ Taipei Times, 3 de julio de 2017.

${ }_{15}^{14}$ Ming Lee, “Taiwan in Trump's Perspective: A Bargaining Chip?”, Asian Policy, núm. 24, 2017, p. 27.

${ }^{15}$ Taipei Times, 4 de julio de 2017.
} 
aprobó un cambio en la política de Estados Unidos para permitir que los barcos de la Marina estadounidense pudieran llegar regularmente a puertos taiwaneses. ${ }^{16}$

El 17 de octubre, el presidente de la Asociación de la Industria de la Defensa de la República de China, Han Pixiang, y el presidente del Consejo de Negocios Taiwán-Estados Unidos, Rupert Hammond-Chambers, firmaron un memorándum de entendimiento para la cooperación en materia de defensa. ${ }^{17}$

Japón ha seguido una política hacia Taiwán en la que enfatiza el fortalecimiento de la relación bilateral. En enero cambió el nombre de su oficina de representación en Taiwán: Oficina de Taipéi de Asociación e Intercambio con Japón, por Asociación de Intercambio Japón-Taiwán. En marzo, el ministro de Estado para Asuntos Internos y Comunicaciones, Akama Jiro, asistió a un evento de turismo en Taipéi en su capacidad oficial, convirtiéndose en el primer funcionario japonés de alto rango que visita Taiwán desde que Japón rompió relaciones con Taiwán en 1972.

Nuevamente, el gobierno de Beijing ha estado siguiendo una línea que tiende a reducir el espacio internacional de Taiwán. El 26 de diciembre de 2016, el gobierno de la RPCh y el de Santo Tomé y Príncipe, un pequeño Estado africano, firmaron un comunicado conjunto anunciando el establecimiento de relaciones diplomáticas. El gobierno taiwanés envió una nota de protesta y rompió relaciones con ese país, con el que había tenido relaciones diplomáticas desde $1997 .^{18}$

Centroamérica ha sido, históricamente, un bastión para Taiwán pues la mayoría de esos países ha afirmado su lealtad a la isla. Empezó a resquebrajarse en 2007, cuando Costa Rica rompió relaciones con ella para establecerlas con la RPCh. La presidenta Cai Yingwen, tomando en cuenta esta situación, decidió realizar un viaje a Centroamérica en el mes de enero de 2017. Visitó Honduras, Guatemala, El Salvador y Nicaragua. Enfatizó en sus discursos en esos países la ayuda financiera que la isla les había otorgado a lo largo de los

${ }^{16}$ Ankit Panda, "Arms to Taiwan and Action Against a Chinese Bank: Is Trump's US-China Honeymoon Over", <http//thediplomat.com/2017/06/arms-to taiwan-and-action-against-a-chinese.bank-istrumps-us-china-honeymoon-over/>. Consultado 3 de julio de 2017.

${ }^{17}$ Noticias de Taiwán, 19 de octubre de 2017.

${ }^{18}$ South China Morning Post, 21 de diciembre de 2016. 
años, la inversión de las compañías taiwanesas en sus territorios, las becas para que sus estudiantes fueran a complementar sus estudios a Taiwán. ${ }^{19}$

El gobierno paraguayo, por su parte, fortaleció su relación comercial con Taiwán al firmar un acuerdo por el cual se reducirán los aranceles para productos como la carne bovina congelada, leche en polvo, jugos de naranja, entre otros. ${ }^{20}$

Un duro golpe para Taiwán fue la ruptura de relaciones con Panamá, país que estableció relaciones diplomáticas con la RPCh en el mes de junio. ${ }^{21}$ El gobierno de Cai declaró que la RPCh estaba usando "el principio de una sola China" para lograr aislar a Taiwán quitándole a sus aliados diplomáticos. Con ello, argumentan, está violando su posición de seguir un desarrollo pacífico, adoptando una actitud "rígida y errónea", está minando el statu quo en el estrecho de Taiwán, lo que advierten, no impedirá que los taiwaneses sigan defendiendo su democracia y su espacio internacional. ${ }^{22}$

En ese sentido fue también el discurso de Cai Yingwen del día nacional. Enfatizó la defensa de la libertad y la democracia de Taiwán. Pidió a los líderes de Beijing un trabajo conjunto para establecer las bases de un nuevo modelo de interacción en el estrecho de Taiwán que permita lograr la paz y la estabilidad a largo plazo. Pero también habló sobre la necesidad de fortalecer el ejército y el sistema militar en general por medio de intercambios técnicos, con el fin de salvaguardar la integridad territorial y las libertades ganadas con la democracia. ${ }^{23}$

El gobierno taiwanés movilizó a sus funcionarios para tratar de detener los avances chinos en Centroamérica. En julio, el gobierno ${ }^{24}$ aprobó la exención de visa para los países de América Latina y el Caribe que son sus aliados. El ministro de Relaciones Exteriores, David Li, y el ministro de Defensa, Feng Shiguan, realizaron por separado un viaje a América Latina. David Li, durante su visita a Belice, afianzó un acuerdo de cooperación con ese país.

\footnotetext{
${ }^{19}$ Taiwan Today, 13 de enero de 2017.

${ }^{20} \mathrm{http}: / /$ www.americaeconomia.com/ con información de REUTERS. 2 de julio de 2017. Consultado el 20 de julio de 2017.

${ }^{21}$ Xinhua, 14 de junio de 2017.

${ }^{22}<$ http://www.mac.gov.tw/en/News_Content.aspx?n=2BA0753CBE348412\&sms=E828F60C4AFB AF90\&s=D9205C4024A19AFD>. Consultado el 3 de agosto de 2017.

${ }^{23}$ The China Post, 10 de octubre de 2017.

${ }^{24}$ Taiwan Today, 18 de septiembre de 2017.
} 
En septiembre, Cai anunció el incremento de inversión taiwanesa y la compra de productos en los países centroamericanos aliados.

El gobierno taiwanés regresó a su política de presión ayudado por sus aliados diplomáticos, para que su caso fuera considerado en la Asamblea de la ONU. En septiembre, Belice, Burkina Faso, El Salvador, Haití, Honduras, Kiribati, Islas Marshall, Nauru, Nicaragua, Palaos, Paraguay, San Cristóbal y Nieves, Santa Lucia, San Vicente y las Granadinas, Islas Salomón, Suazilandia y Tuvalu pidieron en una misiva dirigida al secretario general de Naciones Unidas, Gutierres, que Taiwán fuera incluido en la agenda. Su petición fue rechazada y no fue incluida. ${ }^{25}$

A fines de octubre, Cai Yingwen realizó un viaje a las Islas Marshall, Tuvalu e Islas Salomón, pasando por Hawái y Guam. En las Islas Marshall, Cai anunció que otorgaría exención de visa a los seis países del Pacífico: Kiribati, Nauru, Islas Salomón, Palaos, Tuvalu y las Islas Marshall, aliados de Taiwán. Señaló también que su gobierno desea fortalecer las relaciones y la cooperación con estos países. Con las Islas Marshall ha establecido un programa de cooperación en el tema de salud alimentaria. En su visita a Tuvalu, Cai reafirmó su compromiso de cooperación mantenido en los 38 años de relaciones diplomáticas, en las áreas de agricultura, energía limpia e intercambios culturales. ${ }^{26}$ En las Islas Salomón, Cai enfatizó la necesidad de profundizar la cooperación en la lucha contra el cambio climático.

Para la presidenta de Taiwán es muy importante afianzar las relaciones diplomáticas con los pequeños Estados del Pacífico para evitar que China los presione para romper con la isla y establecer relaciones con Beijing.

Con los países de la ANSEA, dentro del Nuevo Plan de Promoción de la Política hacia el Sur, Cai ha enfatizado los vínculos culturales y académicos. Ha otorgado la exención de visas a sus ciudadanos, becas para sus estudiantes, extensión de permisos de residencia para profesionales y trabajadores técnicos, y un plan de cooperación entre las industrias médicas y farmacéuticas. La administración de Cai también está tratando de obtener el apoyo de la comunidad musulmana de estos países asentada en Taiwán, con planes de establecer lugares

\footnotetext{
${ }^{25}$ Taiwan Today, 28 de septiembre de 2017.

${ }^{26}$ Focus Taiwan, News Channel, 1 de noviembre de 2017, <http://www.focustaiwan.tw>. Consultado el 5 de noviembre de 2017.
} 
en las estaciones de transporte público donde puedan hacer sus oraciones; también promueve la certificación halal dentro de la industria de servicios alimentarios.

En fin, el gobierno del PDP está tratando de evitar que Beijing los aísle. Es un esfuerzo titánico dada la posición de China en el área Asia Pacífico y en la arena internacional.

\section{CONSIDERACIONES FINALES}

Los cambios en el sistema político de Taiwán a lo largo de los años han puesto a la isla en una situación complicada, dada la posición de la RPCh de recuperar los territorios que alguna vez fueron parte del país. Desde el gobierno de Jiang Zemin se hacía apremiante encontrar una solución que llevara a la reunificación. Se percataban de que, entre más tiempo pasara, sería más difícil alcanzarla. Y tenían razón. La sociedad taiwanesa ha cambiado mucho; las nuevas generaciones no están dispuestas a aceptar las imposiciones del continente. Los jóvenes han crecido en un ambiente democrático, muy distinto al de sus padres y abuelos. En las últimas elecciones presidenciales y legislativas participaron activamente para que se escuchara su voz. Desean que Taiwán sea reconocido internacionalmente por su democracia, por su desarrollo económico; y se sienten frustrados al ver los obstáculos puestos por la RPCh, con la que no se sienten identificados.

Independientemente de los problemas internos que prevalecen, de los enfrentamientos entre partidos políticos, del descontento de sectores de la sociedad afectados por las reformas que el gobierno de Cai Yingwen está introduciendo, el sistema político sigue el camino democrático, que se fortalece cada vez más.

\section{BIBLIOGRAFÍA}

Ankit Panda, “Arms to Taiwan and Action Against a Chinese Bank: Is Trump's US-China Honeymoon Over". Disponible en: http//thediplomat.com/2017/06/arms-totaiwan-andaction-against-a-chinese.bank-is-trumps-us-china-honeymoon-over/>. Consultado el 3 de julio de 2017. 
Babones, Salvatore, "Taiwan is at the center of Asia's Economy, but on the margins of its meetings", Disponible en: <http://www.forbes,com>. Consultado el 12 de noviembre de 2017.

Brown, David G., "Governing Taiwan is not easy: President Tsai Ing-wen First Year", Brookings, mayo de 2017. Consultado en: $<$ http://www. Brookings.edu $>$. Consultado el 24 de junio de 2017.

Focus Taiwan, News Channel, 1 de noviembre de 2017. Consultado en: <http://www. focustaiwan.tw>. Consultado el 5 de noviembre de 2017.

Jinping, Xi, "Secure a Decisive Victory in Building a Moderately Prosperous Society in All Respects and Strive for the Great Success of Socialism with Chinese Characteristics for a New Era". Discurso en el Décimo Noveno Congreso del Partido Comunista Chino. 18 de octubre de 2017.

Ming Lee, “Taiwan in Trump's Perspective: A Bargaining Chip?”, Asian Policy, núm. 24, 2017, p. 27.

Noticias de Taiwán, 19 de octubre de 2017.

South China Morning Post, 21 de diciembre de 2016.

South China Morning Post, 25 de agosto de 2017.

South China Morning Post, 4 de septiembre de 2017.

Taipei Times, 19 de octubre de 2017.

Taipei Times, 3 de julio de 2017.

Taipei Times, 4 de julio de 2017.

Taipei Times, 5 de septiembre de 2017.

Taiwan News, 24 de junio de 2017. Disponible en: <http://www.taiwannews.com.tw>. Consultado el 1 de julio de 2017.

Taiwan Today, 13 de enero de 2017.

Taiwan Today, 18 de septiembre de 2017.

Taiwan Today, 28 de septiembre de 2017. 
Taiwan Today, 8 de septiembre de 2017.

The China Post, 10 de octubre de 2017.

Xinhua, 12 de mayo de 2017.

Xinhua, 14 de junio de 2017. 